\title{
Desafios da mobilidade ativa na perspectiva dos serviços públicos: experiências na cidade de São Paulo'
}

\author{
Active Mobility Challenges under public services perspective: experiences in \\ the city of São Paulo
}

Silvia Stuchi Cruz [a] $@$, Sonia Regina Paulino [b]

[a] Universidade de São Paulo (USP), , Escola de Artes, Ciências e Humanidades (EACH), Programa de Pós-
graduação em Sustentabilidade, São Paulo, SP, Brasil.
[b] Universidade de São Paulo (USP), Escola de Artes, Ciências e Humanidades (EACH), São Paulo, SP, Brasil.

Como citar: Cruz, S. S., \& Paulino, S. R. (2019). Desafios da mobilidade ativa na perspectiva dos serviços públicos: experiências na cidade de São Paulo. urbe. Revista Brasileira de Gestão Urbana, v. 11, e20190026. https://doi.org/10.1590/2175-3369.011.e20190026

\section{Resumo}

O trabalho tem o objetivo de analisar os desafios da mobilidade ativa na perspectiva da inovação em serviços. Foram selecionados como estudos de caso: as áreas 40 de São Miguel Paulista e Santana e o projeto de rua completa da Rua Joel Carlos Borges. Na perspectiva da interpretação da inovação em serviços baseada em novas competências e técnicas do prestador, os projetos contribuíram para o desenvolvimento de competências no setor público para utilização de metodologias de avaliação de impacto e metodologia de leitura urbana, trazendo uma visão sistêmica e sobre os usos e dinâmicas locais, e aspectos comportamentais dos usuários. Tais competências estão associadas ao urbanismo tático como técnica para intervenções urbanas temporárias. Os princípios aplicados nos projetos estão alinhados à Política Nacional de Mobilidade Urbana, mas até o momento se restringiram a ações de alteração do limite de velocidade dos veículos e de sinalização horizontal para identificação do perímetro dessas áreas. Não foi identificada introdução de serviços de tratamento cicloviário. Portanto, apontam-se grandes potencialidades para a continuação da introdução de inovações para aprimoramento e ganho de escala dos serviços públicos para transporte não motorizado em consonância com o paradigma da mobilidade urbana sustentável.

Palavras-chave: Mobilidade urbana sustentável. Mobilidade ativa. Urbanismo tático. Inovação.

\section{Abstract}

The goal is to analyze the challenges of active mobility in service innovation context, based on these case studies: São Miguel Paulista and Santana reduced speed zones and Joel Carlos Borges complete street project. From the service innovation perspective based on new competences and techniques of service provider, the case studies contributed to develop public sector competences related to impact evaluation

\footnotetext{
${ }^{1} O$ presente trabalho foi realizado com apoio da Coordenação de Aperfeiçoamento de Pessoal de Nível Superior - Brasil (CAPES) - Código de Financiamento 001.
}

SSC é pós-doutoranda em Sustentabilidade, e-mail: silviastuchi@usp.br

SRP é professora associada, e-mail: sonia.paulino@usp.br 
methodologies and urban reading methodology, resulting in a systemic view on the uses, local dynamics and behavioral aspects of the users. These competences are associated with tactical urbanism practice as a technique for temporary urban interventions. The criteria implemented in the projects are aligned with the National Urban Mobility Policy but are still limited to actions for changing cars speed limit and for horizontal signaling for limiting the perimeter of these areas. No introduction of cycling treatment services has been identified. Therefore, many potential benefits have been listed to carry on with the introduction of innovations for the improvement and scale-up of public services for non-motorized transportation following the paradigm of sustainable urban mobility

Keywords: Sustainable urban mobility. Active mobility. Tactical urbanism. Innovation.

\section{Introdução}

Um dos Objetivos de Desenvolvimento Sustentável (ODS) visa "tornar as cidades e os assentamentos humanos inclusivos, seguros, resilientes e sustentáveis" (United Nations, 2018), estabelecendo metas para a mobilidade urbana, notadamente por meio da expansão dos transportes públicos e com a incorporação do transporte não motorizado, conforme a meta 11.2, que aponta "até 2030, proporcionar o acesso a sistemas de transporte seguros, acessíveis, sustentáveis e a preço acessível para todos, melhorando a segurança rodoviária por meio da expansão dos transportes públicos" (IPEA, 2018, p. 276). Há uma ampla gama de definições para mobilidade urbana sustentável, e a dificuldade de se obter uma definição única deve-se ao fato de que o transporte não pode ser visto de forma isolada dos demais âmbitos da sociedade (Gudmundsson, 2004; Boareto, 2003), o que significa que a sustentabilidade dos sistemas de transportes deve ser considerada como parte das mudanças em todo sistema socioeconômico, levando-se em conta características locais. A mobilidade urbana é sustentável quando visa minimizar e mitigar os impactos ambientais e quando há o acesso universal de toda a população à cidade e oportunidades, contribuindo-se para o desenvolvimento econômico e social (Banister, 2008; Gomide \& Galindo, 2013). No que tange à mobilidade urbana, a ascensão das megacidades coloca grandes desafios de diversas naturezas, que não se restringem aos sistemas de transporte (aumento da motorização, oferta e investimentos no espaço viário), incluindo também aspectos que se referem ao uso e ocupação do solo, tecnológicos, socioeconômicos e ambientais, ou seja, há de se discutir para além do uso do automóvel, alternativas de transporte público e coletivo e o incentivo ao transporte ativo integrado aos demais meios de transporte e para a micromobilidade (deslocamentos a pequenas distâncias) (IPEA, 2016; Brasil, 2018).

Em relação às emissões de gases de efeito estufa (GEE) no Brasil, a atividade agropecuária é a principal emissora, responsável por $74 \%$ das emissões. No entanto, quando se aborda o setor de energia, as atividades de transportes ocupam o primeiro lugar: com 48\% do total das emissões, ou seja, 204 milhões de toneladas emitidas em 2016, distribuídas de modo equivalente nas atividades de transporte de carga e passageiros (Observatório do Clima, 2018). No município de São Paulo, apesar de levar apenas 30\% dos passageiros, os automóveis são responsáveis por 73\% das emissões de GEE (IEMA, 2017). Ressalta-se ainda que, somado ao fato da emissão de gases poluentes, o excessivo e mal planejado uso do transporte motorizado privado também ocupa demasiadamente o espaço urbano, seja pelas vias por onde percorrem, seja pelas vagas de estacionamento, acarretando a degradação da qualidade da vitalidade urbana (Barczak \& Duarte, 2012).

Para reverter esse cenário, necessita-se de políticas e medidas que melhorem as alternativas ao automóvel, aprimorando a infraestrutura e os serviços relacionados ao transporte ativo e ao transporte público coletivo. Esses desafios devem ser abordados combinando mudanças nos parâmetros ambientais e urbanos, enfatizando a combinação de transporte público e transporte ativo (não motorizado), como a mobilidade de pedestres e ciclistas. Para tanto, ressalta-se o papel dos mecanismos de governança participativa nos países em desenvolvimento, buscando envolver os cidadãos nas tomadas de decisão 
sobre a distribuição de fundos públicos entre comunidades, conformação de políticas públicas, bem como no acompanhamento e avaliação das despesas governamentais.

A Política Nacional de Mobilidade Urbana PNMU (Lei 12.587/Brasil, 2012a) define diretrizes, atribuindo prioridade aos meios de transporte não motorizados e ao serviço público coletivo de transporte. Considera-se que esta é a primeira lei nacional que aborda a temática da mobilidade de modo mais abrangente na perspectiva da equidade, sustentabilidade e participação da sociedade (ANTP, 2017), por meio de órgãos colegiados, ouvidorias nas instituições responsáveis pela gestão do Sistema Nacional de Mobilidade Urbana, audiências e consultas públicas; e procedimentos sistemáticos de comunicação, de avaliação da satisfação dos cidadãos e dos usuários e de prestação de contas públicas (Brasil, 2012a). No entanto, apesar das estratégias ambiciosas apontadas pela PNMU, observa-se no Brasil uma clara tendência de incremento de vendas de veículos motorizados individuais, por meio de políticas públicas e incentivos voltados ao setor automotivo. Como exemplos, o programa Inovar Auto, que vigorou de 2012 a 2017, de incentivo à inovação tecnológica e adensamento da cadeia produtiva de veículos automotores, implantado pelas leis no 12.715/2012 (Brasil, 2012b) e no 12.996/2014 (Brasil, 2014); e o Programa Rota 2030, que fomenta a mobilidade elétrica e os biocombustíveis no Brasil, com a previsão de incentivos fiscais de até $\mathrm{R} \$ 1,5$ bilhões por ano a empresas automobilísticas que investirem no mínimo $\mathrm{R} \$ 5$ bilhões em P\&D de biocombustíveis no Brasil. Ou seja, os resultados positivos da PNMU dependerão de uma interação que envolve diferentes organismos, instituições e pessoas. Desse modo, para haver transformações efetivas na mobilidade urbana, é imprescindível um esforço político para promover as diversas formas de integração e melhorar os processos de governança (Machado \& Piccinini, 2018).

Para os fins da PNMU, no Quadro 1 são apresentadas as definições de sistema de transporte, sistema nacional de mobilidade urbana, transporte urbano e mobilidade; e a classificação dos serviços de transporte urbano, no que tange ao modo de transporte, objeto e natureza do serviço:

Quadro 1 - Sistema Nacional de Mobilidade Urbana - definições e classificações

\begin{tabular}{|c|c|c|c|}
\hline $\begin{array}{l}\text { Definição de Sistema de } \\
\text { Transporte }\end{array}$ & \multicolumn{3}{|c|}{$\begin{array}{l}\text { "as partes que os compõem são as vias, os veículos, os terminais que se interagem de modo } \\
\text { a promover deslocamento espacial de pessoas e mercadorias" (Kawamoto, 2004). }\end{array}$} \\
\hline $\begin{array}{c}\text { Definição de Sistema } \\
\text { Nacional de Mobilidade } \\
\text { Urbana } \\
\end{array}$ & \multicolumn{3}{|c|}{$\begin{array}{l}\text { "conjunto organizado e coordenado dos modos de transporte, de serviços e de } \\
\text { infraestruturas que garante os deslocamentos de pessoas e cargas no território do } \\
\text { Município" (Brasil, 2012a, Art. } 3^{\circ} \text { ) }\end{array}$} \\
\hline $\begin{array}{l}\text { Definição de Transporte } \\
\text { Urbano }\end{array}$ & \multicolumn{3}{|c|}{$\begin{array}{l}\text { "conjunto dos modos e serviços de transporte público e privado utilizados para o } \\
\text { deslocamento de pessoas e cargas nas cidades integrantes da Política Nacional de } \\
\text { Mobilidade Urbana" (Brasil, 2012a, Art. } 4^{\circ} \text { I) }\end{array}$} \\
\hline $\begin{array}{l}\text { Definição de Mobilidade } \\
\text { Urbana }\end{array}$ & \multicolumn{3}{|c|}{$\begin{array}{l}\text { "condição em que se realizam os deslocamentos de pessoas e cargas no espaço } \\
\text { urbano" (Brasil, 2012a, Art. } 4^{\circ} \text { II) }\end{array}$} \\
\hline \multirow{3}{*}{ Classificação } & Modos de transporte & Objeto & Natureza do serviço \\
\hline & - motorizados & - de passageiros & - público \\
\hline & - não motorizados & - de cargas & - privado \\
\hline
\end{tabular}

Fonte: elaboração própria. Grifo dos autores.

A partir das informações no Quadro 1 e das disposições do Art. 6‥ II e IV da PNMU (Brasil, 2012a), é possível traçar a relação entre mobilidade urbana sustentável e transporte ativo, destacando os desafios na prestação de serviços públicos. Especificamente sobre a mobilidade urbana, identifica-se um conjunto de serviços e meios de transporte de pessoas e carga, assim como as interações entre esses deslocamentos e o meio urbano. Ou seja, o termo mobilidade urbana vem sobrepondo o termo transporte por sua definição mais ampla, abarcando, para além dos sistemas de transporte, o planejamento urbano, a disponibilidade e a disposição de bens e serviços na cidade e o acesso a eles (Gomide \& Galindo, 2013; Barbosa, 2016). 0 transporte não motorizado/ativo é definido como "qualquer forma de transporte humano, como caminhar, pedalar, cadeira de rodas, com o auxílio de muletas, enfim, todos os deslocamentos feitos de forma autônoma pelos cidadãos, mesmo com o uso de dispositivos auxiliares" (Secretaria Nacional de Transporte e da Mobilidade Urbana, 2007, p. 88). Dessa forma, desempenha um 
papel importante no contexto urbano, pois promove a inclusão social e o desenvolvimento urbano equitativo, ou seja, é um integrante importante da mobilidade urbana sustentável.

Isto posto, este artigo investiga como os serviços públicos podem ser melhorados para ampliar a mobilidade ativa em contextos de megacidades. 0 objetivo é analisar os desafios da mobilidade ativa na cidade de São Paulo, utilizando a abordagem da inovação em serviços. O Manual de Oslo (OCDE, 2018) apresenta a definição geral de inovação, que é aplicável a todos os setores da economia (Negócios, Governo, Instituições sem fins lucrativos): "inovação é um produto ou processo novo ou melhorado (ou combinação deles) que difere significativamente dos produtos ou processos anteriores da unidade e que tem sido disponibilizado a potenciais utilizadores (produto) ou posto em uso (processo) pela unidade ${ }^{2 "}$ (tradução livre OCDE, 2018, p. 20). Indo além dos fatores puramente tecnológicos, as inovações não tecnológicas organizacionais, relacionais, sociais, entre outras (Djellal et al., 2013) - possuem papel relevante para análise da inovação em serviços públicos. Nesse sentido, propõe que um serviço pode ser definido como a mobilização de competências internas ou externas e técnicas internas ou externas (tangíveis ou intangíveis) para produzir as características finais do produto (bem ou serviço) (Gallouj \& Weinstein, 1997; Gallouj, 2002). E a prestação do serviço é marcada pela ocorrência de atividades, sendo que:

[...] uma atividade de serviço é uma operação destinada a provocar uma mudança no estado da realidade de $\mathrm{C}$ que é de propriedade ou utilizada pelo consumidor $\mathrm{B}$, a alteração efetuada pelo prestador de serviços A a pedido de B e, em muitos casos, em colaboração com ele ou ela, mas não induzindo à produção de um bem que circula na economia independentemente de C (tradução livre de Gadrey, 2000, p. 375).

São considerados três casos na cidade de São Paulo - Área 40 de São Miguel Paulista; Área 40 de Santana e Rua Completa da Joel Carlos Borges: Santana, por ter passado da etapa de intervenção temporária a permanente, São Miguelpor contemplar a via com maior número de mortes de pedestres na capital. Já para o projeto de Rua Completa, a iniciativa da Rua Joel Carlos Borges, zona sul de São Paulo, é a única existente na cidade. As iniciativas selecionadas incluem a perspectiva multi/intermodal proposta na PNMU, incluindo acesso à rede de transporte público - ônibus e trens.

No projeto de área 40, delimita-se um perímetro onde a velocidade máxima permitida é de $40 \mathrm{~km} / \mathrm{h}$, com o objetivo de melhorar a segurança do sistema viário de usuários vulneráveis, pedestres e ciclistas, visando à redução de ocorrências e melhoria da qualidade ambiental (CET, 2016; ITDP, 2016; ITDP, 2018). 0 projeto de Rua Completa é baseado na distribuição equitativa do espaço, o que proporciona segurança e conforto a todas as pessoas, de todas as idades, utilizando todos os meios de transporte. Além disso, ao melhorar as condições de mobilidade ativa, as ruas completas e a zona de velocidade reduzida diminuem os níveis de ruído, melhoram a qualidade urbana e, por possivelmente tornar o local mais atrativo para a mobilidade ativa e acesso ao transporte público coletivo, melhoram a qualidade do ar e reduzem as emissões de gases de efeito estufa (WHO, 2018; ITDP, 2018; Urb-i, 2019; WRI, 2018). Nos três casos, são apresentadas práticas de urbanismo tático, ou seja, ações temporárias realizadas em determinado território para testar esses espaços a diferentes usos que posteriormente podem vir a receber a intervenção permanente. Para Cloutier et al. (2018), essas intervenções contribuem para a criação de uma narrativa cívica em favor da adaptação à mudança climática em escala local, buscando promover reestruturações de base, de modo participativo, sendo uma forma de reapropriação do espaço urbano por seus próprios usuários. A metodologia de pesquisa proposta baseia-se em cinco etapas: identificação de iniciativas de mobilidade ativa; mapeamento dos projetos em cada iniciativa; seleção dos casos estudados; coleta de dados primários e secundários: relatórios e entrevistas; e aplicação do modelo da inovação em serviços baseado em características.

\footnotetext{
${ }^{2}$ "An innovation is a new or improved product or process (or combination thereof) that differs significantly from the unit's previous products or processes and that has been made available to potential users (product) or brought into use by the unit (process)" (OCDE, 2018, p. 20).
} 
Após a introdução, a seção 2 aborda a mobilidade ativa e as megacidades; a seção 3 apresenta o referencial teórico; a seção 4 mostra a metodologia; por fim, nas seções 5 e 6 são apresentados os resultados e as considerações finais, respectivamente.

\section{A mobilidade ativa e as megacidades}

Podem ser destacadas várias áreas prioritárias de ação política para a promoção de transporte urbano mais sustentável nos países em desenvolvimento: transporte público rodoviário; transporte público ferroviário; transporte não motorizado; e melhoria tecnológica (Pojani \& Stead, 2018, p. 91). Isso pode ser mais bem abordado por meio de políticas e programas de transporte apropriados usando intervenções baseadas em evidências, que exigem ampla compreensão dos padrões de mobilidade urbana.

No caso das megacidades nos países em desenvolvimento, Zhao (2010) aponta que a questão central a ser abordada é a expansão urbana sustentável, que requer medidas para gerenciar o desenvolvimento metropolitano. Um dos passos importantes nessa direção é promover o transporte sustentável. Cidades com mais de 10 milhões de habitantes são denominadas megacidades (UN, 2018) e, para além da quantidade populacional, essa classificação está associada também à urbanização, fator considerado forte promotor de impactos ambientais.

A mobilidade urbana sustentável é representada pelo deslocamento de pessoas e bens no espaço urbano não apenas por veículos motorizados, mas também por modos não motorizados (Gudmundsson, 2004; Boareto, 2003). A maioria dos brasileiros e brasileiras se deslocam de modos ativos, e há também uma porcentagem expressiva de viagens por transporte público coletivo. Em 2016, 43\% das viagens foram realizadas a pé e por bicicleta; $29 \%$ por transporte individual motorizado; e $28 \%$ por transporte público coletivo. 0 uso do transporte público consome $33 \%$ da energia total em $28 \%$ do total das viagens realizadas (ANTP, 2018). 0 aprimoramento e a ampliação territorial dessa divisão modal para cidades maiores podem ser um caminho no sentido de estabilizar as emissões de gases de efeito estufa, no que tange ao setor de transportes (Barczak \& Duarte, 2012). Dados os grandes desafios impostos às megacidades, as soluções de mobilidade urbana sustentável necessitam também estar conectadas às questões-chave da governança urbana, à política habitacional, uso e ocupação do solo, ao saneamento e aos planos diretores (Di Giulio et al., 2018). A Figura 1 destaca alguns dados importantes sobre o contexto da mobilidade urbana em megacidades do Brasil:

\begin{tabular}{|l|l|}
\hline $\begin{array}{l}208 \text { milhões habitantes: a população } \\
\text { brasileira é de } 208 \text { milhões habitantes, com } \\
85 \% \text { das pessoas vivendo em áreas urbanas }\end{array}$ & $\begin{array}{l}\text { As duas megacidades do país são São Paulo e Rio de } \\
\text { Janeiro que juntas representam 17\% do total da população } \\
\text { urbana do Brasil e respondem por 25,5\% do PIB }\end{array}$ \\
\hline \begin{tabular}{|l|l|} 
R\$98 bilhões: os congestionamentos nas \\
cidades brasileiras geram ainda grandes \\
prejuízos econômicos
\end{tabular} & $\begin{array}{l}\text { Os custos dos congestionamentos nas duas principais } \\
\text { regiöes metropolitanas do pais (SP e RJ) em 2013 } \\
\text { ultrapassou R\$ } 98 \text { bilhões, valor equivalente a 2\% do PIB }\end{array}$ \\
\hline $\begin{array}{l}\mathbf{8 0 \%} \text { do espaço viário: de acordo com a } \\
\text { demanda apresentada na divisão modal, há } \\
\text { um desequilíbrio na utilização do espaço } \\
\text { viário }\end{array}$ & $\begin{array}{l}80 \% \text { do espaço viário em São Paulo é destinado ao } \\
\text { transporte individual motorizado }\end{array}$ \\
\hline
\end{tabular}

Figura 1 - Características da mobilidade urbana em megacidades do Brasil. Fonte: elaboração própria com base em IBGE (2010), Firjan (2014), FGV (2018), ANTP (2018), IEMA (2017).

Nesse sentido, a PNMU estabelece que os Planos Municipais de Mobilidade (PlanMobs) devem priorizar pedestres e bicicletas (modos não motorizados), em vez de veículos motorizados, e transporte público coletivo em vez de transporte motorizado individual, mitigando-se assim os custos ambientais e as externalidades negativas sociais e econômicas decorrentes dos serviços de mobilidade urbana (Brasil, 2012a). Atingir esse cenário é um grande desafio, uma vez que em muitas cidades brasileiras prevalece 
uma combinação de arranjos administrativos frágeis, limitada capacidade de planejamento e falta de coordenação entre uso da terra e formulação de políticas de transporte, que juntos inibem o desenvolvimento de projetos mais inovadores, políticas integradas e sustentáveis (Machado \& Piccinini, 2018). Além disso, os PlanMobs não estão integrados a um planejamento de transportes urbano e metropolitano mais abrangentes e têm se mostrado desconectados das realidades locais (Barbosa, 2016; Machado \& Piccinini, 2018), inclusive nas duas megacidades brasileiras.

A pesquisa centra-se na cidade de São Paulo / Brasil, que possui pouco mais de 12 milhões de habitantes (IBGE, 2017), sendo o maior conglomerado urbano da América do Sul, com grau de urbanização de 99,1\% (SEADE, 2018). Apenas $25 \%$ da população tem acesso próximo -num raio de até $1 \mathrm{~km}$ de distância - a uma estação de transporte público coletivo (metrô, trem, BRT e VLT) (ITDP, 2016; WRI, 2016). Em São Paulo predomina a sensação de insegurança das pessoas quando se deslocam a pé pela cidade (CET, 2016). Em pesquisa realizada em 2018, 88\% dos entrevistados afirmam que se sentem "pouco seguros" ou "nada seguros" quando se deslocam a pé por São Paulo. Na mesma pesquisa, 30\% dos paulistanos afirmaram que, se fossem tomadas medidas de segurança para a circulação dos ciclistas, utilizariam a bicicleta como meio de transporte (Rede Nossa São Paulo, 2018).

Sobre recentes marcos regulatórios municipais, o Conselho Municipal de Transporte e Trânsito da cidade de São Paulo foi criado em julho de 2013 (São Paulo, 2013) com caráter consultivo, propositivo e participativo, com câmaras temáticas criadas de forma bottom-up, que emergiram a partir da solicitação da sociedade civil (Tartarotti \& Merchán, 2017). As câmaras temáticas de bicicleta e mobilidade a pé foram criadas em 2015. No mesmo ano, a comissão técnica de Mobilidade a Pé e Acessibilidade da ANTP encabeçou a elaboração de uma proposta de diretrizes para a mobilidade a pé no município (que foram parcialmente incorporadas ao PlanMob do município de São Paulo). Em setembro de 2016, foi instituído o Programa Bike SP para incentivar o uso de bicicleta como meio de transporte. Em junho de 2017, foi aprovado o Estatuto do Pedestre da cidade de São Paulo (Lei no 16.673, São Paulo, 2017). Nos exemplos aqui citados, destaca-se o papel da sociedade civil através de organizações sem fins lucrativos, contribuindo para atingir esses objetivos.

A integração da mobilidade ativa com o transporte público como um campo para inovação em serviços públicos.

De modo geral, quando se aborda a questão da inovação vinculada a questões urbanas, vem à tona as "cidades inteligentes", definidas como aquelas que se utilizam de tecnologias da informação e comunicação para transformar e melhorar a disponibilidade e a qualidade das infraestruturas e serviços públicos, a diminuição do hiato entre poder público e cidadãos (e-democracia, e-governo/governo digital), os padrões de organização, aprendizagem, gerenciamento das infraestruturas a todos os atores, tendo como pano de fundo o desenvolvimento sustentável. Sua definição é ampla e descreve várias perspectivas (econômica, ambiental, social e econômica), visando melhorar a qualidade de vida dos cidadãos (Artmann et al., 2019), ou seja, também vinculada à mobilidade urbana sustentável e promoção da mobilidade ativa.

Desse modo, a incorporação de conceitos e políticas mais amplas de inovação é importante para que se explore o potencial da inovação em serviços, no intuito de lidar com (e dar conta de) mobilidade e transporte. Esse tema é um importante impulsionador da inovação e confirma que os impactos da inovação vão muito além do conceito tradicional de "competitividade" para incluir também problemas ambientais e sociais (Stare, 2013). Trata-se de tema de natureza complexa, multiforme, sistêmica e muitas vezes conflitante (Djellal \& Gallouj, 2015), logo, não pode ser resolvido por um único ator - a inovação em serviços, por definição, se baseia em interações entre diferentes atores (Stare, 2013). Stare (2013) e Cruz \& Paulino (2013) mostram que os desafios são ainda maiores em contextos das economias em desenvolvimento e emergentes, em um contexto de inovação substancialmente marcado pelo olhar da inovação tecnológica. Nas economias emergentes, os serviços públicos demandam inovações não apenas em termos de aumento de eficiência, mas também em relação à transparência e à capacidade de resposta às necessidades dos usuários/cidadãos (Mohnen \& Stare, 2013). 
O setor público é também um sistema de serviços em que as inovações podem melhorar o desempenho dessas atividades e, por fim, incidir na qualidade de vida dos cidadãos. Parte da natureza multifacetada e heterogênea do setor público é resultante de suas várias interfaces, tais como: 1) a sua interface com o setor privado; 2) a interface entre o setor público e os cidadãos, e 3) interfaces internas no setor público (Bugge et al., 2010). Embora existam as modalidades de transferência da produção do serviço público, o setor público, como responsável pela provisão do serviço, deve assegurar que esta produção seja realizada de modo adequado.

Uma vez que diferentes tipos de atores estão envolvidos no processo de inovação, a prestação de serviços em uma configuração multiagente permite o desenvolvimento de complementaridades e sinergias em meio aos diferentes agentes, cada uma com seus próprios objetivos e competências específicas (Windrum \& García-Goñi, 2008; Desmarchelier et al., 2018). No campo de serviços, há ainda avanços no sentido de compreender as especificidades dos serviços públicos e os contextos socioeconômicos em que são prestados. As configurações multiagentes em serviços públicos abordam agentes públicos, privados, sociedade civil e cidadãos, para coproduzir inovações, de várias naturezas: novo serviço, novas formas de organização, novos processos, métodos, e combinação das anteriores. Nesse contexto, a participação das partes interessadas torna-se essencial. Ademais, a área de estudo sobre inovação nos serviços públicos possibilita a inclusão mais evidente e palpável de formas não tecnológicas de inovação (Desmarchelier et al., 2018).

Os serviços baseados na integração da mobilidade ativa e do transporte público dependem das condições do ambiente de caminhada e para pedestres e ciclistas, que podem ser mais bem entendidas a partir da abordagem de inovação de serviços públicos. Desse modo, a abordagem adotada no presente trabalho é a integradora, perspectiva mais abrangente para explicar inovação em serviços, fornecendo uma estrutura ampla abordando inovações tecnológicas e não tecnológicas, bem como os diversos atores que participam do serviço em questão. As inovações não tecnológicas - organizacionais, relacionais e sociais (Djellal et al., 2013; Desmarchelier et al., 2018) - possuem papel relevante para análise da inovação em serviços públicos. Para tanto, utiliza-se o Modelo de representação do produto e da inovação em termos de características do serviço, adotando-o como uma abordagem multiagentes. De acordo com Gallouj \& Weinstein (1997) e Gallouj (2002), o produto (seja bem ou serviço) é entendido a partir de um conjunto de vetores de características e competências, representadas no modelo baseado em características. O modelo é atualizado em Gallouj \& Savona (2010), acrescentando - para além das técnicas dos prestadores - as técnicas do cliente e, mais recentemente, Djellal et al. (2013) adicionam/sobrepõem vetores buscando integrar os diferentes agentes. Como demonstrado pela Figura 2, $[\mathrm{Y}]$ corresponde às características do serviço, [T] às características técnicas internas; [T'] às características técnicas externas; $[\mathrm{C}]$ às competências internas; $\mathrm{e}\left[\mathrm{C}^{\prime}\right]$ às competências externas. 


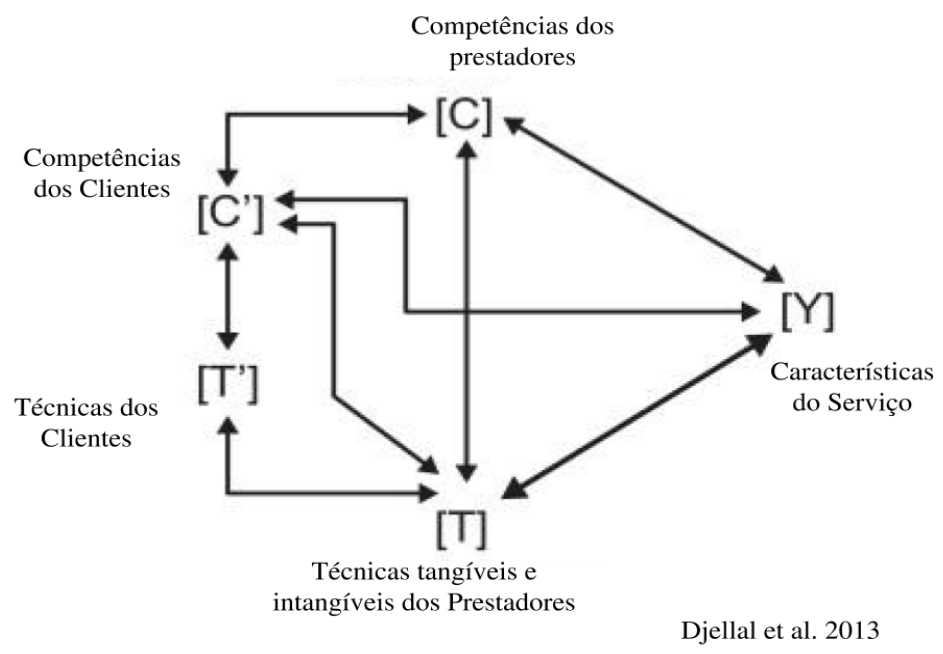

Figura 2 - O produto (bem ou serviço) como um conjunto de características, técnicas e competências. Fonte: Djellal et al. (2013).

O vetor de características técnicas dos prestadores ( $\mathrm{T}$ ) e usuários diretos ( $\left.\mathrm{T}^{\prime \prime}\right)$ é o conjunto de técnicas utilizadas para realizar o produto (bem ou serviço). Técnicas tangíveis (material de informática, máquinas, equipamentos, e outros itens de infraestrutura) ou intangíveis (métodos matemáticos, métodos de trabalho). As técnicas são codificadas, transmissíveis e independentes dos indivíduos. 0 vetor competências dos prestadores (C) e usuários ( $\left.\mathrm{C}^{\prime \prime}\right)$ é o conjunto de competências mobilizadas para realizar o serviço, que podem ser científicas, técnicas, operacionais e relacionais. São mobilizadas para tornar acessível a utilização das técnicas ou a realização do serviço. As competências são incorporadas aos indivíduos, a um grupo de indivíduos ou a uma organização, e decorrem da formação, experiência, interações etc. São tácitas e dificilmente transmissíveis. Uma contribuição ao modelo original foi feita pelos autores Windrum \& García-Goñi (2008), sugerindo a inclusão do governo no modelo de Gallouj \& Weinstein (1997), apontando que a qualidade de um bem ou serviço é resultado de um conjunto de competências detidas por provedores/produtores e pelos usuários. Destacam-se as competências dos provedores de serviços, que são classificadas em back office competences e user facing competences, ou competências que são necessárias na interação com os usuários. Assim, quando tratamos da inovação em serviços prestados pelo setor público, no caso serviços de mobilidade urbana, devemos fazer um esforço para o entendimento da relação de serviço entre prestador e usuário, uma vez que a concepção de Gadrey (2000) e outros autores que abordam a inovação em serviços trata dos serviços marcados pelo relacionamento próximo entre cliente/usuário e prestador de serviço. Desse modo, destaca-se que cada tipo de serviço se relaciona inteiramente com as competências necessárias para a sua execução. Sendo que nos serviços que possuem pouco contato entre prestador e usuário do serviço, as competências exigidas são, basicamente, competências do prestador.

\section{Metodologia}

A metodologia de pesquisa proposta tem como recorte geográfico-temporal a cidade de São Paulo, e o período considerado para a coleta de dados é de 2015 (por conta do PlanMob de SP) até janeiro de 2019; e baseia-se em cinco etapas mostradas no Quadro 2 
Quadro 2 - Metodologia de pesquisa proposta

\begin{tabular}{|c|c|c|c|c|}
\hline $\mathbf{1}$ & $\mathbf{2}$ & $\mathbf{3}$ & $\mathbf{4}$ & $\mathbf{5}$ \\
\hline $\begin{array}{c}\text { Identificação de } \\
\text { iniciativas de } \\
\text { mobilidade ativa }\end{array}$ & $\begin{array}{c}\text { Mapeamento dos } \\
\text { projetos em cada } \\
\text { iniciativa }\end{array}$ & $\begin{array}{c}\text { Seleção dos casos } \\
\text { estudados: áreas 40 } \\
\text { de São Miguel } \\
\text { Paulista e Santana; } \\
\text { e rua completa Joel } \\
\text { Carlos Borges }\end{array}$ & $\begin{array}{c}\text { Coleta de dados } \\
\text { primários e } \\
\text { secundários: relatórios } \\
\text { e entrevistas }\end{array}$ & $\begin{array}{c}\text { Aplicação do modelo da } \\
\text { inovação em serviços } \\
\text { baseado em } \\
\text { características }\end{array}$ \\
\hline$\rightarrow$ & $\rightarrow$ & $\rightarrow$ & $\rightarrow$ & \\
\hline
\end{tabular}

Fonte: elaboração própria.

\section{Identificação de iniciativas de mobilidade ativa}

São Paulo é uma das 10 cidades selecionadas para o programa Iniciativa Global de Segurança Rodoviária Bloomberg Philanthropies (Iniciativa Bloomberg), que visa reduzir os ferimentos e fatalidades resultantes de colisões em todo o mundo, contemplando as 10 cidades (Accra, Adis Abeba, Bandung, Bangcoc, Bogotá, Fortaleza, Ho Chi Minh, Mumbai, São Paulo e Xangai) em dez países: Brasil, Camboja, China, Egito, Índia, Quênia, México, Rússia, Turquia e Vietnã. Como parte do programa, vem sendo desenvolvidos os projetos de Área 40 e Ruas Completas. Inicialmente, realizou-se um mapeamento das iniciativas na cidade de São Paulo.

\section{Mapeamento dos projetos em cada iniciativa}

O Quadro 3 apresenta as informações sobre as iniciativas de Áreas 40 e Rua Completa mapeadas na cidade de São Paulo.

Quadro 3 - Mapeamento de projetos Áreas 40 e Rua Completa em São Paulo

\begin{tabular}{|c|c|c|}
\hline Área 40 & Implantação & Zona \\
\hline Vias da Bela Vista & Abr/2016 & Centro \\
\hline Rótula Central - $1^{a}$ Fase & Out/ 2013 & Centro \\
\hline Rótula Central - $2^{a}$ Fase & Dez/2014 & Centro \\
\hline Rótula Consolação & Jun/2015 & Centro \\
\hline Região da Lapa - $1^{a}$ Fase & Set/2014 & Oeste \\
\hline Região da Lapa - $2^{a}$ Fase & Mar/2015 & Oeste \\
\hline Santana & Set/2014 & Norte \\
\hline Moema $-1^{a}$ Fase & Nov/2014 & Sul \\
\hline Moema - $2^{a}$ Fase & Nov/2014 & Sul \\
\hline Penha & Dez/2014 & Leste \\
\hline Brás & Fev/2015 & Leste \\
\hline São Miguel Paulista & Set/2015 & Leste \\
\hline Rua Completa & Implantação & Zona \\
\hline Rua Joel Carlos Borges & Set/2017 & Sul \\
\hline
\end{tabular}

Fonte: Elaboração própria com base em CET, 2019.

\section{Seleção dos casos estudados: Áreas 40 de São Miguel Paulista e Santana e Rua Completa Joel Carlos Borges}

A seleção dos casos estudados utilizou os seguintes critérios:

- Desenvolvido na cidade de São Paulo; 
- Participação Multiagente;

- Aderentes à Política Nacional de Mobilidade Urbana;

- Consideração de benefícios para a qualidade de vida: redução de poluentes; redução de poluição sonora; redução de acidentes com pedestres e ciclistas.

Das 12 ações mapeadas nos projetos Áreas 40, dois casos foram selecionados: Santana, por ter passado da etapa de intervenção temporária à permanente; São Miguel, por contemplar a via com maior número de mortes de pedestres na capital. Já para o projeto de Rua Completa, a iniciativa da Rua Joel Carlos Borges, zona sul de São Paulo, é a única existente na cidade. As iniciativas selecionadas incluem a perspectiva multi/intermodal proposta na PNMU, incluindo acesso à rede de transporte público - ônibus e trens. No contexto abordado, as partes interessadas são: prefeitura - representada pela Secretaria Municipal de Mobilidade e Transporte e subprefeituras; empresa mista - Companhia de Engenharia de Tráfego - CET; e organizações da sociedade civil envolvidas com (os) projeto(s).

\section{Coleta de dados primários e secundários: relatórios e entrevistas}

As fontes de dados secundárias utilizadas sobre os casos estudados são: relatórios e diagnósticos realizados por ITDP, FGV, WRI, Cidade Ativa, Metrópole 1:1; Urb-i e Labmob-UFRJ; banco de dados da CET (2019) de Áreas 40 e Programa de Proteção à Vida - PPV. Para a coleta de dados primários, foram aplicados questionários semiestruturados com sete agentes: representantes de organizações da sociedade civil (WRI; ITDP e Iniciativa Bloomberg), Start up (Urb-i), universidades participantes dos diagnósticos de avaliação de impacto (FGV e LabMob/ UFRJ) e poder público (SMT). O conjunto de dados obtidos é explorado com a aplicação do modelo baseado em características para interpretação da inovação em serviços e análise de conteúdo. Com base em Bardin (1977), o processo da análise de conteúdo da aplicação das entrevistas com os atores-chave e materiais coletados relacionados aos projetos fundamentou-se na organização da análise, fase em que foi realizada uma pré-análise e leitura inicial dos relatórios e diagnósticos e, na etapa de exploração do material, foram selecionados os materiais a serem considerados para estudo e quais partes dos materiais seriam estudadas. 0 enfoque foi direcionado para descrição dos projetos, modificações nos serviços pré e pós a implantação dos projetos; participação das partes interessadas, atores envolvidos, desenvolvimento de novas tecnologias e metodologias pertinentes à mobilidade ativa, capacitação de corpo técnico, novas competências e técnicas adquiridas, e melhorias ambientais. A categorização realizada é resultante da sistematização e análise dos dados a partir dos vetores Caraterísticas do Serviço (Y), Técnicas (T) e Competências (C) dos agentes envolvidos - que permitiu o detalhamento de técnicas e competências internas ou externas (tangíveis ou intangíveis) para compreender as características do serviço estudado.

\section{Aplicação do modelo baseado em características para interpretação da inovação em serviços}

Seguido do processo da análise de conteúdo da aplicação das entrevistas com os atores-chave e materiais secundários coletados, a avaliação dos aspectos inovadores introduzidos no serviço público para a mobilidade ativa baseia- se no modelo de representação do produto e da inovação baseado nas características do serviço (Gallouj, 2002; Gallouj \& Savona, 2010; Djellal et al., 2013). 


\section{Experiências de mobilidade ativa em são paulo a partir da prestação de serviços públicos}

\section{Caracterização dos projetos estudados}

Os Quadros 4 e 5 apresentam dados das iniciativas estudadas, salientando o tipo de projeto voltado à mobilidade ativa, localização, ano de implantação (temporária e permanente, quando for o caso) e breve descrição.

Quadro 4 - Informações sobre os projetos estudados

\begin{tabular}{|c|c|c|}
\hline Projeto & Localização & Ano de Implantação \\
\hline Rua Completa & Rua Joel Carlos Borges & 2017 \\
\hline \multicolumn{3}{|c|}{$\begin{array}{l}\text { Breve descrição: O projeto, ao melhorar as condições de mobilidade ativa, as ruas completas e a zona de velocidade } \\
\text { reduzida, diminui os níveis de ruído, melhora a qualidade urbana, e por possivelmente tornar o local mais atrativo para } \\
\text { a mobilidade ativa e acesso ao transporte público coletivo, melhora a qualidade do ar e reduz as emissões de gases } \\
\text { com efeito estufa. }\end{array}$} \\
\hline
\end{tabular}

Fonte: elaboração própria.

Quadro 5 - Informações sobre os projetos estudados

\begin{tabular}{|c|c|c|c|c|}
\hline \multirow{2}{*}{ Projeto } & Localização & Ano de implantação & $\begin{array}{c}\text { Intervenção urbana } \\
\text { temporária }\end{array}$ & $\begin{array}{c}\text { Intervenção urbana } \\
\text { permanente }\end{array}$ \\
\hline \multirow{2}{*}{ Área 40 } & $\begin{array}{c}\text { São Miguel Paulista, } \\
\text { Zona Leste }\end{array}$ & 2015 & 2016 & - \\
\cline { 2 - 5 } & Santana, Zona Norte & 2014 & 2017 & 2018 \\
\hline
\end{tabular}

Breve descrição: Os projetos de Área 40 das regiões de São Miguel Paulista e Santana apresentam um histórico de elevados índices de atropelamentos. Por isso, um perímetro delimitado como área de velocidade reduzida (Área 40) foi implantado em 2014 e 2015, respectivamente. A regulamentação da redução da velocidade per si não costuma ser suficiente para garantir a efetividade desta medida. Para tanto, necessita-se intervir também na geometria viária, tornando-a mais segura para pedestres e ciclistas, por exemplo, por meio de elementos de moderação de tráfego. O desenvolvimento do projeto de redesenho urbano e segurança viária de São Miguel Paulista ocorreu sob a coordenação da Iniciativa Bloomberg. O projeto insere-se no planejamento das intervenções nas Áreas 40, da CET. Em novembro de 2016, São Miguel Paulista recebeu uma intervenção urbana temporária de um dia que simulou uma proposta de novo desenho viário, permitindo que a circulação de pedestres existente fosse feita de forma contínua e segura, diminuindo o conflito com veículos motorizados e melhorando a visibilidade de todos os usuários da via. A partir da intervenção de São Miguel Paulista, surge a oportunidade de replicar o projeto em outra área da cidade, na Zona Norte, em Santana. Em Santana, o projeto temporário recebeu adaptações e, em 2018, recebeu a implantação permanente. As intervenções não visam interferir na quantidade de usuários, mas garantir maior segurança aos pedestres e ciclistas que circulam nessas localidades.

Fonte: elaboração própria.

\section{Aspectos inovadores do serviço público destinado à mobilidade ativa}

Os casos estudados demonstram inovação nos serviços prestados pela prefeitura basicamente no tratamento da infraestrutura viária para pedestres. Os aspectos inovadores introduzidos no serviço público para a mobilidade ativa podem ser interpretados a partir da aplicação do modelo da inovação em serviços baseado em características, visualizada na Figura 3. 


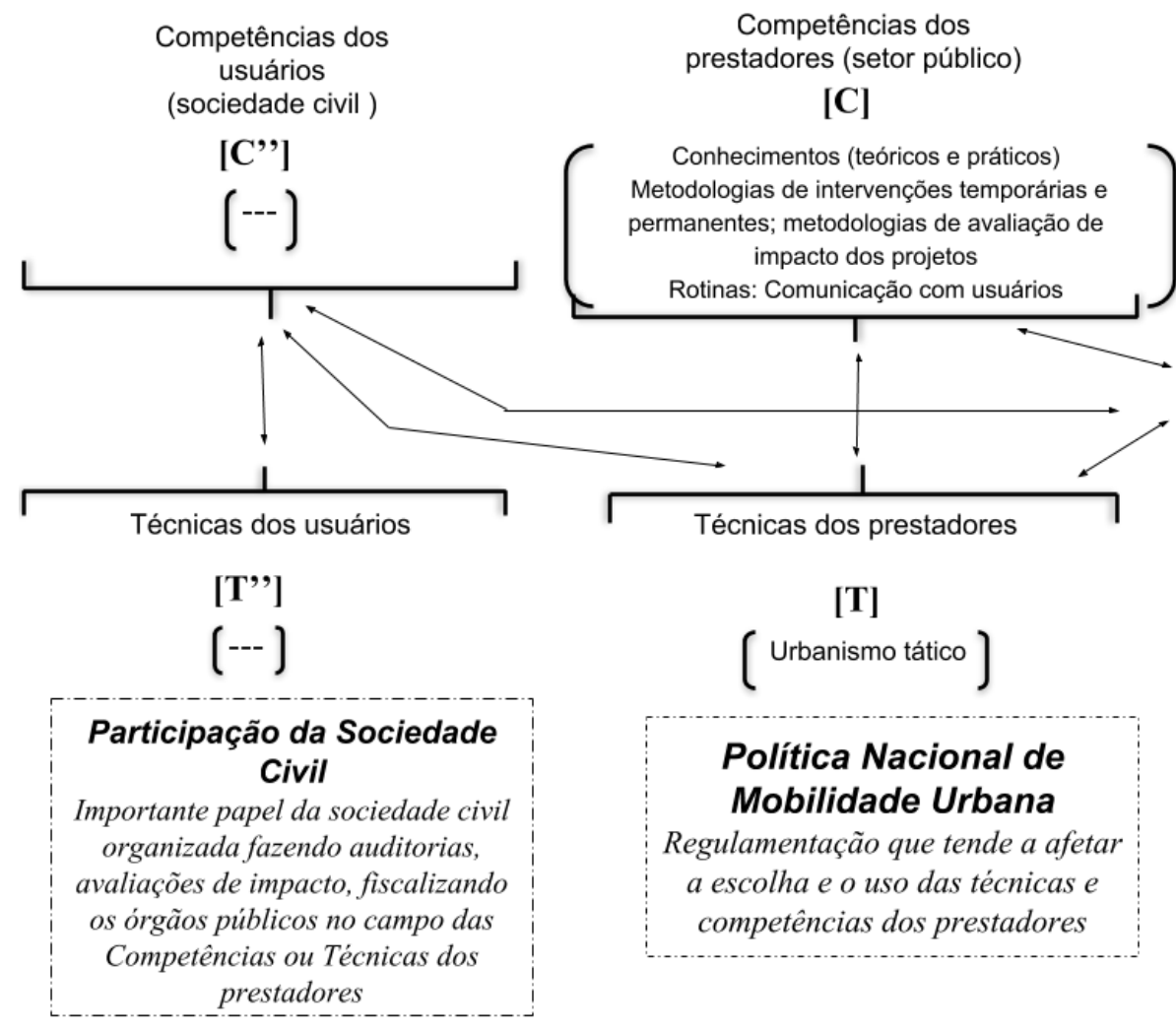

Características dos Serviços

\section{$[\mathbf{Y}]$}

Tratamento da infraestrutura viária para pedestres

Figura 3 - Modelo baseado em características para interpretação da inovação em serviços adaptado para os casos estudados. Fonte: elaboração própria.

\section{Participação da sociedade}

Nos casos estudados, destaca-se o importante papel da sociedade civil organizada fazendo auditorias, avaliações de impacto, fiscalizando os órgãos públicos no campo das competências $(\mathrm{C})$ ou técnicas dos prestadores (T). A prestação de serviços públicos geralmente envolve uma gama mais complexa e ampla de atores e instituições. A relação de serviços entre produtor/provedor e usuário amplia-se, incluindo a organização responsável pela prestação do serviço e todo o sistema de regulação e instituições envolvidas (Djellal \& Gallouj, 2008; Desmarchelier et al., 2018). Nos casos estudados, os atores envolvidos são apresentados no Quadro 6:

Quadro 6 - Atores envolvidos nos casos estudados

\section{Área 40 São Miguel Paulista}

Setor público: CET, Secretaria Municipal de Mobilidade e Transporte, Subprefeitura de São

Miguel, SPTrans, SPUrbanismo, SIURB/SPObras. Transporte. Área $\mathbf{4 0}$ Santana

Rua Completa J. C. Borges

Setor público: CET, Subprefeitura de Santana/Tucuruvi, Secretaria Municipal de Mobilidade e Setor privado: Start up Urb-i

ade civil: Iniciativa de Políticas de Transporte e Desenvolvimento Bloomberg, Iniciativa Global de (ITDP), Iniciativa Global de Desenho de Desenho de Cidades (NACTOCidades (NACTO-GDCI), WRI Brasil, Vital GDCI), WRI Brasil, Vital Strategies. Strategies.

No estudo de avaliação de impacto: Setorpúblico: CET, SecretaniaMunicipal Nos estudos de avaliação de Fundação Getúlio Vargas (FGV/CEPESP) e de Mobilidade e Transporte, ITDP. Subprefeitura de Pinheiros.
Sociedade civil: WRI impacto: Cidade Ativa, Urb-i, WRI, Labmob UFRJ, Metrópole 1:1

Fonte: elaboração própria. 


\section{Área 40 - São Miguel Paulista}

Em 2016, foi realizado um encontro sobre "desenho e segurança viária" com cerca de 100 participantes em uma apresentação aberta ao público. Após, uma oficina reuniu 23 especialistas e técnicos para colaborarem com o projeto de requalificação urbana e segurança viária da Área 40 de São Miguel Paulista, que foi lançado pela prefeitura no mesmo ano, com o objetivo de testar os princípios do projeto para a Área 40 de São Miguel junto aos moradores da área de intervenção temporária.

\section{Área 40 - Santana}

Em 2017, a etapa de exploração de soluções para os conflitos identificados incluiu encontros com a população da região, com o objetivo de identificar prioridades e demandas para o entorno dos pontos de intervenção e também ampliar o entendimento sobre segurança no trânsito, desenho urbano e moderação de tráfego e conceitos de urbanismo tático e intervenção urbana temporária. A ação foi inicialmente apresentada em uma palestra com cerca de 70 participantes. Após a intervenção temporária, a prefeitura realizou a obra permanente em 2018 e, agora, trabalha no diagnóstico de avaliação de impacto dessa intervenção.

\section{Rua completa - Joel Carlos Borges}

Em 2017, sugeriu-se a realização de workshops com a comunidade antes da implementação do projeto temporário, com o objetivo de informar a população sobre a intervenção e coletar possíveis sugestões. O projeto foi implantado sem uma comunicação clara com a população (Guido et al., 2017; Urb-i, 2019). Nesse sentido, em novembro de 2017 foram aplicados questionários para investigar a percepção e a avaliação de comerciantes das ruas e pessoas que passam no local sobre o impacto do projeto de rua completa e para investigar hábitos sobre transporte e consumo de pessoas que acessam o local. A maioria (92\%) dos passantes aprovou a iniciativa (WRI, 2018; Cidade Ativa, 2018b).

\section{Características do serviço de mobilidade ativa $(\mathrm{Y})$}

No vetor de características dos serviços $(\mathrm{Y})$ de manutenção e requalificação de infraestrutura para o modo a pé e a integração com o transporte público, foi identificada a introdução de novos tipos de tratamento da infraestrutura viária para pedestres: de calçadas; de travessias; de acessibilidade; de sinalização horizontal e vertical no espaço público; de sistema semafórico adequado para transporte não motorizado; e de moderação de tráfego. Não foram identificados novos tipos de tratamento cicloviário. Visando melhorar as condições de integração com o transporte público coletivo, as iniciativas estudadas buscaram aumentar segurança e conforto dos pedestres a partir da redução de velocidade, da reforma e ampliação de calçadas.

De modo específico para cada iniciativa estudada, verificou-se que, na Área 40 de São Miguel Paulista, estão localizados uma estação da CPTM e calçadões de comércio popular e de rua. 0 tráfego de veículos nessa região é intenso, são mais de 50 linhas municipais de ônibus que passam no local, compostas por uma variedade de comportamentos de fluxos, com diversos pontos de origem e destino. Contudo, as condições de infraestrutura para pedestres e ciclistas são precárias, e na área localiza-se a $4^{\text {a }}$ via com mais atropelamentos fatais em 2017. A intervenção temporária criou uma via exclusiva para circulação de pedestres e, a partir de intervenções físicas permanentes no viário da Área 40 de São Miguel Paulista, espera-se que haja uma melhoria nas condições de circulação a pé nesta região (Cidade Ativa, 2018a; FGV, 2017a; FGV, 2017b; ITDP, 2016). 
Para o caso de Santana, no centro da Área 40 estão localizados uma estação do metrô e um terminal de ônibus. No total, circulam 87 linhas de ônibus, que passam em quase todas as ruas da localidade, nos seis trechos de faixas de ônibus. Há também três trechos de ciclovias. Na intervenção de 2017, pinturas temporárias ampliaram ilhas de refúgio, deixando mais espaço para os pedestres que aguardam para atravessar, e alargaram calçadas em todas as esquinas, duas vagas de estacionamento se tornaram espaços de convívio (parklets) e uma rotatória foi incluída. Com isso, houve uma diminuição da velocidade dos veículos e um encurtamento das travessias. Com a intervenção temporária proposta em 2017, aumentou-se em 40\% da área para os pedestres (ITDP, 2018). As transformações realizadas foram pontuais e têm implicação mais direta na micromobilidade, mas o fato de estarem em área de abrangência do transporte coletivo amplifica seu impacto. 0 projeto permanente foi implantado em 2018.

Na rua completa Joel Carlos Borges localiza-se o acesso a uma estação de trem. A conexão com o trem permite que a iniciativa faça parte de viagens de maiores distâncias, considerando a intermodalidade (WRI, 2018). No local não há infraestrutura específica para bicicleta. Dado o alto fluxo de pedestres, a implantação das faixas verdes com 1,5 metro a 2,00 metros de largura, demarcação com balizadores, tachas refletivas e vasos de plantas aumentou de $29 \%$ para $70 \%$ o espaço viário designado para os caminhantes (Guido et al., 2017; WRI, 2018; Cidade Ativa, 2018b; Urb-i, 2019). Após as modificações, $80 \%$ das pessoas que passam pela Rua Joel a consideraram mais segura. Ainda assim, foi possível notar que muitas pessoas caminham pela via, principalmente nos horários de pico de entrada e saída do trabalho e almoço (WRI, 2018; Cidade Ativa, 2018b; Urb-i, 2019). Esse cenário é um indicativo da vocação da via: ser voltada exclusivamente a pedestres e veículos não motorizados, permitindo o fluxo restrito de veículos motorizados para acesso aos edifícios ao longo da via.

\section{Competências do prestador de serviço (C)}

As competências dos prestadores diretos (C) referem-se a conhecimentos (teóricos e práticos) e rotinas. Destacam-se os conhecimentos necessários à utilização de metodologias de avaliação de impacto de intervenções urbanas favoráveis à mobilidade ativa e ampliação do escopo de metodologia de leitura urbana. Os projetos ultrapassam os limites habituais de escopo da CET (contagens volumétricas, sinalização e operação viária). Para viabilizar a adoção prática de urbanismo tático nos casos estudados, a implantação dos projetos foi acompanhada do desenvolvimento de novas competências. Nos três casos, observa-se a tentativa de estabelecer novas rotinas de comunicação com usuários, com o desenvolvimento de oficinas de capacitação e coleta de ideias e as intervenções temporárias, que buscam, além de testar a transformação de determinados espaços públicos, também coletar dados sobre a percepção dos usuários em relação ao projeto proposto. Os encontros foram coordenados por organizações da sociedade civil, com apoio do poder público. Além disso, nos casos de São Miguel Paulista e da Rua Joel Carlos Borges, ocorreu a aplicação de metodologia de estudo observacional de avaliação de impacto dos projetos, a escore de propensão (propensity score matching), que objetiva avaliar os impactos de uma intervenção por meio da comparação de grupos: o que recebe a intervenção (factual) e o grupo que não é alterado (contrafactual). Nos casos estudados (factuais), os contrafactuais (áreas de controle) são apresentadas no Quadro 7:

Quadro 7 - Factuais e Contrafactuais dos estudos de avaliação de impacto

\begin{tabular}{cc}
\hline Factual & Contrafactual (área de controle) \\
\hline São Miguel Paulista & Região no Entorno do Mercado da Lapa \\
Santana & Não houve estudo de avaliação de impacto \\
Joel Carlos Borges & Rua Gomes Carvalho, ao lado da Estação Vila Olímpia. \\
\hline
\end{tabular}


Sobre a metodologia de contagem, tradicionalmente a CET realiza contagens volumétricas veiculares e de pedestres. A metodologia de leitura urbana empregada nos casos estudados vai além do âmbito quantitativo e realiza também: levantamentos geométricos; mapeamento de atividades de permanência; observações comportamentais, tais como travessias na faixa e fora da faixa de pedestre, pessoas caminhando fora da calçada em trechos de estrangulamento, garantindo-se que os principais aspectos que precisam ser reformados, adaptados ou construídos, sejam identificados e analisados. Ainda, contempla-se a realização de entrevistas ex-ante e ex-post às intervenções (temporárias e permanentes), no intuito de avaliar a percepção dos usuários em relação ao redesenho da geometria viária, à segurança e conforto, às interferências nos deslocamentos e aos impactos econômicos no comércio local.

\section{Técnicas do prestador do serviço (T)}

As técnicas dos prestadores diretos (T) são relacionadas à introdução de práticas de urbanismo tático (intervenções temporárias) realizadas em determinado território para testar esses espaços a diferentes usos e que, posteriormente, podem vir a receber a intervenção permanente. Essas intervenções, de curto prazo e baixo custo, buscam promover reestruturações de base, de modo participativo, sendo uma forma de reapropriação do espaço urbano por seus próprios usuários. De modo geral, o urbanismo tático se distribui em etapas de observação dos usos existentes, observação comportamental dos usuários e dinâmica do espaço, contemplando medições e contagens de tráfego, pedestres e ciclistas.

No entanto, verificou-se que não foi introduzida a medição de indicadores para avaliar a contribuição potencial dessas iniciativas de mobilidade ativa na redução da poluição sonora, da poluição do ar e na política municipal de mudanças climáticas. Apenas no caso de São Miguel, a avaliação de impacto da iniciativa incluiu inicialmente a avaliação da qualidade do ar por meio de um minissensor móvel, denominado Bicycle Environmental Mapping -BeMap (FGV, 2017b). 0 equipamento pode ser acoplado ao guidão de uma bicicleta para mensurar níveis de monóxido de carbono (CO) e óxidos de nitrogênio (NOx), além de dados de temperatura e umidade. A coleta de dados pelo BeMap foi uma aplicação-piloto e pioneira para fins de pesquisa. Há a grande vantagem de ser móvel e, portanto, tem o potencial de produzir mapas de poluição do ar das áreas estudadas. No entanto, problemas técnicos com os sensores e com o manuseio do aparelho impediram a coleta de dados de poluição do ar, prejudicando a avaliação. Como alternativa, por meio de uma parceria com o laboratório da Faculdade de Medicina da USP, foram instaladas estações fixas para coleta de material particulado (MP2,5), que coletaram amostras de MP2,5 durante três semanas. No entanto, por serem estações fixas, não possuem a capacidade de captar variações de poluição do ar por toda a extensão da área 40 (FGV, 2017b).

\section{Considerações finais}

De acordo com as etapas metodológicas propostas, visando analisar os desafios da mobilidade ativa na perspectiva da inovação em serviços, a partir da identificação e mapeamento das iniciativas de mobilidade ativa na cidade de São Paulo, foram selecionados como estudos de caso: as Áreas 40 de São Miguel Paulista e Santana e o projeto de rua completa da Rua Joel Carlos Borges. Nos casos estudados, salienta-se a majoritária aprovação por parte dos usuários, o que é um desdobramento importante da inovação representada pela introdução de serviços públicos de tratamento da infraestrutura viária para pedestres, com destaque para o papel da sociedade civil organizada fazendo auditorias, avaliações de impacto, fiscalizando os órgãos públicos no campo das competências ou técnicas dos prestadores.

A aplicação do modelo da inovação em serviços baseado em características aos casos estudados demonstra que a inovação nos serviços prestados pela prefeitura ocorreu basicamente no tratamento da infraestrutura viária para pedestres. Na perspectiva da interpretação da inovação em serviços baseada em novas competências e técnicas do prestador, os projetos contribuíram para o desenvolvimento de 
competências no setor público para utilização de metodologias de avaliação de impacto de intervenções urbanas favoráveis à mobilidade ativa, e metodologia de leitura urbana, para além das contagens de veículos, pedestres e ciclistas, trazendo uma visão sistêmica e sobre os usos e dinâmicas locais, e aspectos comportamentais dos usuários. Tais competências estão associadas ao urbanismo tático como técnica para intervenções urbanas temporárias.

Os princípios aplicados nos casos estudados estão alinhados à Política Nacional de Mobilidade Urbana, mas até o momento se restringiram a ações para alteração do limite de velocidade dos veículos e para sinalização horizontal para identificação do perímetro dessas áreas. Não foi identificada introdução de serviços de tratamento cicloviário. Portanto, apontam-se grandes potencialidades para a continuação da introdução de inovações para aprimoramento e ganho de escala dos serviços públicos para transporte não motorizado em consonância com o paradigma da mobilidade urbana sustentável.

Recomenda-se a busca pela consolidação e expansão dos projetos de ruas completas e Áreas 40, dando suporte às intervenções (temporárias e permanentes) estabelecidas que alteram a prioridade viária, destinando aos usuários mais vulneráveis porções mais adequadas do espaço urbano, tornando os locais mais atrativos para a mobilidade ativa e acesso ao transporte público coletivo, melhorando a qualidade do ar e reduzindo emissões de gases de efeito estufa. De modo geral, verificou-se certa resistência política para implementar projetos que visem priorizar a mobilidade ativa, tanto dentro do governo como entre parte da população, em especial por se tratar de mudanças que exigem retirada de espaço do automóvel (principalmente vagas de estacionamento) ou redução significativa de velocidade. Algumas vezes essa resistência se origina do medo do desconhecido, e o urbanismo tático colabora para quebrar essa resistência, permitindo a vivência e experimentação do espaço de forma temporária - o redesenho pode ser removido ou ajustado caso o resultado não seja positivo.

Por fim, dado que os projetos ainda são relativamente recentes e abrangem um recorte geográficotemporal específico, ainda é necessário um amadurecimento dos resultados advindos dos casos estudados, bem como o aprofundamento da análise de projetos da mesma natureza em outros contextos urbanos e sociais.

\section{Referencias}

Artmann, M., Kohler, M., Meinel, G., Gan, J., \& Ioja, I. (2019). How smart growth and green infrastructure can mutually support each other: A conceptual framework for compact and green cities. Ecological Indicators, 96, 1022. http://dx.doi.org/10.1016/j.ecolind.2017.07.001.

Associação Nacional de Transportes Público - ANTP. (2017). Mobilidade humana para um brasil urbano. São Paulo: ANTP. Recuperado em 01 de junho de 2019, de http://files.antp.org.br/2017/7/12/antp-mobilidade-humana-1107-2017--baixa.pdf

Associação Nacional de Transportes Público - ANTP. (2018). Sistema de informações da mobilidade urbana. São Paulo: ANTP. Recuperado em 01 de junho de 2019, de http://www.antp.org.br/sistema-de-informacoes-damobilidade/apresentacao.html

Banister, D. (2008). The sustainable mobility paradigm. Transport Policy, 15(2), 73-80.

http://dx.doi.org/10.1016/j.tranpol.2007.10.005.

Barbosa, A. S. (2016). Mobilidade urbana para pessoas com deficiência no Brasil: um estudo em blogs. urbe. Urbe. Revista Brasileira de Gestão Urbana, 8(1), 142-154. http://dx.doi.org/10.1590/2175-3369.008.001.A003.

Barczak, R., \& Duarte, F. (2012). Impactos ambientais da mobilidade urbana: cinco categorias de medidas mitigadoras. urbe. Urbe. Revista Brasileira de Gestão Urbana, 4(1), 13-32. http://dx.doi.org/10.1590/S217533692012000100002.

Bardin, L. (1977). Análise de conteúdo. Lisboa: Edições 70.

Boareto, R. A. (2003). Mobilidade urbana sustentável. Revista dos Transportes Públicos, 25(100), 45-56. 
Brasil. (2012a, 03 de janeiro). Lei 12.587/12, de 03 de janeiro de 2012. Estabelece a Política Nacional De Mobilidade Urbana. Brasília: Diário Oficial.

Brasil. (2012b, 17 de setembro). Lei 12.715/12, de 17 de setembro de 2012. Institui o Programa de Incentivo à Inovação Tecnológica e Adensamento da Cadeia Produtiva de Veículos Automotores. Brasília: Diário Oficial.

Brasil. (2014, 18 de junho). Lei 12.996/14, de 18 de junho de 2014. Altera a Lei no 12.715, de 17 de setembro de 2012, que institui o Programa de Incentivo à Inovação Tecnológica e Adensamento da Cadeia Produtiva de Veículos Automotores - INOVAR-AUTO. Brasília: Diário Oficial.

Brasil. (2018). Plano de ação em ciência, tecnologia e inovação para ciências humanas e sociais. Brasília: Centro de Gestão e Estudos Estratégicos.

Bugge, M., Hauknes, J., Bloch, C., Slipersæter, S. (2010). Measuring public innovation in the nordic countries: toward a common statistical approach. Aarhus: The Danish Centre for Studies in Research and Research Policy.

Cidade Ativa. (2018a). Diagnóstico áreas 40: São Miguel Paulista. São Paulo: Cidade Ativa. Recuperado em 01 de junho de 2019, de www.cidadeativa.org

Cidade Ativa. (2018b). Diagnóstico ruas completas: Joel Carlos Borges. São Paulo: Cidade Ativa. Recuperado em 01 de junho de 2019, de www.cidadeativa.org

Cloutier, G., Papin, M., \& Bizier, C. (2018). Do-it-yourself (DIY) adaptation: civic initiatives as drivers to address climate change at the urban scale. Cities, 74, 284-291. http://dx.doi.org/10.1016/j.cities.2017.12.018.

Companhia de Engenharia de Tráfego - CET. (2016). De modal de transporte à satisfação de andar a pé. (Boletim 59). São Paulo: CET. Recuperado em 01 de junho de 2019, http://www.cetsp.com.br/media/507576/bt59.pdf

Companhia de Engenharia de Tráfego - CET. (2019). Área 40: área de velocidade reduzida. São Paulo: Secretaria Municipal de Mobilidade e Transportes. Recuperado em 01 de junho de 2019, de http://www.cetsp.com.br/consultas/seguranca-e-mobilidade/area-40.aspx

Cruz, S., \& Paulino, S. (2013). Analysis of access to clean development mechanism landfill projects through a multiagent model. International Journal of Environmental Sciences and Development, 4(3), 268-271.

http://dx.doi.org/10.7763/IJESD.2013.V4.351.

Desmarchelier, B., Djellal, F., \& Gallouj, F. (2018, 20-22 September). Services in innovation networks and innovation networks in services: from Traditional Innovation Networks (TINS) to Public Service Innovation Networks (PSINS). In Proceedings of The 28th RESER Conference (pp. 1-19). Gothenburg: RESER.

Di Giulio, G. M., Bedran-Martins, A. M. B., Vasconcellos, M. P., Ribeiro, W. C., \& Lemos, M. C. (2018). Mainstreaming climate adaptation in the megacity of São Paulo, Brazil. Cities, 72, 237-244.

http://dx.doi.org/10.1016/j.cities.2017.09.001.

Djellal, F., \& Gallouj, F. (2008). Measuring and improving productivity in services: issues, strategies and challenges. Cheltenham: Edward Elgar.

Djellal, F., \& Gallouj, F. (2015). Quinze avancées et quinze défis pour la recherche sur l'innovation dans service, ClerséCNRS. France: Lille 1 University.

Djellal, F., Gallouj, F., \& Miles, I. (2013). Two decades of research on innovation in services: which place for public services? Structural Change and Economic Dynamics, 27, 98-117. http://dx.doi.org/10.1016/j.strueco.2013.06.005.

Federação das Indústrias do Estado do Rio de Janeiro - FIRJAN. (2014, julho). Os custos da (i)mobilidade nas regiões metropolitanas do Rio de Janeiro e São Paulo (Nota Técnica, No. 3). Rio de Janeiro: FIRJAN. Recuperado em 01 de junho de 2019, de https://www.firjan.com.br/publicacoes/publicacoes-de-economia/os-custos-da-imobilidade-nas-regioes-metropolitanas-do-rio-de-janeiro-e-sao-paulo.htm

Fundação Getúlio Vargas - FGV. (2017a). Relatório de desenho de pesquisa para avaliação de impacto do Projeto de Requalificação Urbana e Segurança Viária de São Miguel Paulista na poluição do ar e na saúde. São Paulo: FGV.

Fundação Getúlio Vargas - FGV. (2017b). Relatório de linha de base da avaliação de impacto do projeto de requalificação urbana e segurança viária de São Miguel Paulista. São Paulo: FGV. 
Fundação Getúlio Vargas - FGV. (2018). Gastos públicos em mobilidade urbana no Brasil, centro de estudos em sustentabilidade. São Paulo: FGVces. Recuperado em 01 de junho de 2019, de http://mediadrawer.gvces.com.br/publicacoes-2/original/fgvces_gastospublicosmobilidade_jun-18.pdf

Gadrey, J. (2000). The characterization of goods and services: an alternative approach. Review of Income and Wealth, 46(3), 369-387.

Gallouj, F. (2002). Innovation in the service economy: the new wealth of nations (vol. 32). Cheltenham: Edward Elgar. http://dx.doi.org/10.4337/9781843765370.

Gallouj, F., \& Savona, M. (2010). Towards a theory of innovation in services: a state of the art. In F. Gallouj, \& F. Djellal. (Eds.), The handbook of innovation and services (pp. 27-48). Edward Elgar Publishing. https://doi.org/10.4337/9781849803304.

Gallouj, F., \& Weinstein, O. (1997). Innovation in services. Research Policy, 26(4-5), 537-556. http://dx.doi.org/10.1016/S0048-7333(97)00030-9.

Gomide, A. Á., \& Galindo, E. P. (2013). A mobilidade urbana: uma agenda inconclusa ou o retorno daquilo que não foi. Estudos Avançados, 27(79), 27-39. http://dx.doi.org/10.1590/S0103-40142013000300003.

Gudmundsson, H. (2004). Sustainable transport and performance indicators. In R. E. Hester \& R. M. Harrison (Eds.), Transport and the environment (Issues in Environmental Science and Technology, no. 20, pp. 35-63). Cambridge: Royal Society of Chemistry.

Guido, C., Franco, P., Fogelson, Y. (2017). A urgência das transformações urbanas. O case da rua Joel Carlos Borges como ponto de partida para políticas de reequilíbrio e valorização do espaço público. Revista dos Transportes Públicos - ANTP. Recuperado em 01 de junho de 2019, de http://www.antp.org.br/revista-dos-transportespublicos/apresentacao.html

Institute for Transportation and Development Policy - ITDP. (2016). Desenho urbano e segurança viária: requalificação de áreas de baixa velocidade em São Miguel Paulista. Brasília: ITDP.

Institute for Transportation and Development Policy - ITDP. (2018). Intervenção urbana temporária: (re)pensando a rua em Santana (Relatório de Atividade). Brasília: ITDP.

Instituto Brasileiro de Geografia e Estatística - IBGE. (2010). Censo 2010 - População residente por situação de domicílio. Rio de Janeiro: IBGE. Recuperado em 01 de junho de 2019, de

https://www.ibge.gov.br/estatisticas/sociais/habitacao/9662-censo-demografico-2010.html?=\&t=destaques

Instituto Brasileiro de Geografia e Estatística - IBGE. (2017). Estimativas populacionais dos municípios para 2017. Rio de Janeiro: IBGE. Recuperado em 01 de junho de 2019, de https://ww2.ibge.gov.br/home/estatistica/populacao/estimativa2017/default.shtm

Instituto de Energia e Meio Ambiente - IEMA. (2017). Inventário de emissões do transporte rodoviário de passageiros em São Paulo. São Paulo: IEMA. Recuperado em 01 de junho de 2019, de http://emissoes.energiaeambiente.org.br/

Instituto de Pesquisa Econômica Aplicada - IPEA. (2016). Cidade e movimento: mobilidades e interações no desenvolvimento urbano (1. ed.) Brasília: Institute for Transportation and Development Policy.

Instituto de Pesquisa Econômica Aplicada - IPEA. (2018). Agenda 2030: ODS - Metas Nacionais dos objetivos de desenvolvimento sustentável. Brasília: IPEA. Recuperado em 01 de junho de 2019, de http://www.ipea.gov.br/portal/images/stories/PDFs/livros/livros/180801_ods_metas_nac_dos_obj_de_desenv_s usten_propos_de_adequa.pdf

Kawamoto, E. (2004). Análise de sistemas de transporte. 2. ed. São Carlos: Universidade de São Paulo.

Machado, L., \& Piccinini, L. S. (2018). Os desafios para a efetividade da implementação dos planos de mobilidade urbana: uma revisão sistemática. Urbe. Revista Brasileira de Gestão Urbana, 10(1), 72-94.

http://dx.doi.org/10.1590/2175-3369.010.001.ao06.

Mohnen, P., \& Stare, M. (2013). The notion of inclusive innovation. In W. Wobbe (Ed.), Inclusive innovation and service innovation (Policy Brief, no. 15). Luxembourg: Publications Office of the European Union. 
Observatório do Clima. (2018). Emissões de GEE no Brasil e suas implicações para políticas públicas e a contribuição brasileira para o Acordo de Paris. São Paulo: SEEG. Recuperado em 01 de junho de 2019, de http://seeg.eco.br/wpcontent/uploads/2018/08/Relatorios-SEEG-2018-Sintese-FINAL-v1.pdf

Organização de Cooperação e de Desenvolvimento Económico - OCDE. (2018). Oslo manual 2018 guidelines for collecting - Reporting and using data on innovation (4th ed.). Paris: OCDE.

Pojani, D., \& Stead, D. (2018). Policy design for sustainable urban transport in the global south. Policy Design and Practice, 1(2), 90-102. http://dx.doi.org/10.1080/25741292.2018.1454291.

Rede Nossa São Paulo. (2018). Viver em São Paulo - Mobilidade urbana na cidade. São Paulo: Rede Nossa São Paulo.

São Paulo. Prefeitura Municipal. (2013, 01 de julho). Decreto Municipal n. 54.058, de 01 de julho de 2013. Cria o Conselho Municipal de Trânsito e Transporte - CMTT, no âmbito da Secretaria Municipal de Transportes. São Paulo: Diário Oficial da Cidade de São Paulo, página 01.

São Paulo. Prefeitura Municipal. (2017, 13 de junho). Lei no. 16.673, de 13 de junho de 2017. Estatuto do Pedestre do município de São Paulo. São Paulo: Diário Oficial.

Seade. (2018). Perfil dos municípios paulistas. São Paulo: Fundação Seade. Recuperado em 01 de junho de 2019, de http://www.perfil.seade.gov.br/

Secretaria Nacional de Transporte e da Mobilidade Urbana. (2007). Planmob - caderno de referência para elaboração de plano de mobilidade urbana. Brasília: Ministério das Cidades. Recuperado em 01 de junho de 2019, de http://www.capacidades.gov.br/biblioteca/detalhar/id/270/titulo/planmob---caderno-de-referencia-paraelaboracao-de-plano-de-mobilidade-urbana

Stare, M. (2013). Seizing the opportunities of service innovation. In W. Wobbe (Ed.), Inclusive innovation and service innovation (Policy Brief, no. 7). Luxembourg: Publications Office of the European Union.

Tartarotti, R., \& Merchán, C. R. (2017). Uma análise sobre a incidência do Conselho Municipal de Transporte e Trânsito na política de mobilidade urbana da cidade de São Paulo. In Anais do Congreso Internacional en Gobierno, Administración y Políticas Públicas. Madrid: Espanha.

United Nations. (2018). Sustainable development goals. New York: UN. Recuperado em 01 de junho de 2019, de https://www.un.org/sustainabledevelopment/sustainable-development-goals

Urb-i. (2019). Relatório pós intervenção plano de bairro - Berrini - Rua Joel Carlos Borges × Rua Sansão. São Paulo: Urb-i.

Windrum, P., \& García-Goñi, M. (2008). A neo-Schumpeterian model of health services innovation. Research Policy, Elsevier, 37(4), 649-672. http://dx.doi.org/10.1016/j.respol.2007.12.011.

World Health Organization - WHO. (2018). Global status report on road safety. Geneva: WHO. Recuperado em 01 de junho de 2019, de https://apps.who.int/iris/bitstream/handle/10665/276462/9789241565684-eng.pdf?ua=1

World Resources Institute - WRI. (2016). Infográfico PNT People Near Transit da cidade de São Paulo. Brasília: ITDP. Recuperado em 01 de junho de 2019, de http://wricidades.org/research/publication/infogr\%C3\%A1ficopnt

World Resources Institute - WRI. (2018, março). Estudo de impacto e avaliação de rua completa - Rua Joel Carlos Borges, Berrini - São Paulo (Relatório Técnico Final). Rio de Janeiro: Laboratório de Mobilidade Sustentável LabMob.

Zhao, P. (2010). Sustainable urban expansion and transportation in a growing megacity: consequences of urban sprawl for mobility on the urban fringe of Beijing. Habitat International, 34(2), 236-243. http://dx.doi.org/10.1016/j.habitatint.2009.09.008.

Editor: Janaína Pasqual Lofhagen.

Recebido: Maio 06, 2019

Aprovado: Maio 19, 2019 